\title{
24. The power of visualization choices: Different images of patterns in space
}

\author{
Britta Ricker, Menno-Jan Kraak, and Yuri Engelhardt
}

\begin{abstract}
Maps are representations of the world. They offer summaries or simplifications of data that are collected, attempt to reveal unknowns, to simplify and communicate complex spatial phenomena. Numerous decisions are made in the process of creating a map. Seemingly inconsequential variations of cartographic design decisions offer many ways to illustrate this process. We use an open dataset related to the United Nations Gender Inequality Index to demonstrate design decision points and their output. As governments are increasingly making data open to the public, and map-making tools and software are now more accessible online, these considerations are important both for those making and reading maps online.
\end{abstract}

Keywords: Cartography; SDGs; Open data,

\section{Introduction}

Cartography is defined as the art, science, and technology of making and using maps. It requires both qualitative and quantitative methodologies associated with data handling and information communication design. Maps are often seen as authoritative representations of truth (Pickles, 1995; Wood, 1992). There are interrelated processes, interactions, and negotiations during the design and the data collection phases of map-making (Pavlovskaya, 2018). When designed effectively, maps and diagrams can tell stories, offer interactive, dynamic insights into geographical patterns at multiple scales, and show trends over various temporal scales. A map should help the user to quickly grasp a concept or an idea.

Engebretsen, M. and H. Kennedy (eds.), Data Visualization in Society. Amsterdam: Amsterdam University Press, 2020 DOI 10.5117/9789463722902_CH24 
Maps can be powerful communication tools to convey the distribution and the magnitude of challenges society faces. Increasingly, people with access to the internet also have access to data that are collected by governments and online data visualization. These data could be used to make maps to lead advocacy efforts linked to gender inequality and feminist discourse, but the creation and use of maps for such efforts are not straightforward. Design defaults or visualization constraints in common mapping software packages and Application Programming Interfaces (API) can impose unintended communication consequences.

In this chapter, we pose the following research questions: What are significant cartographic design decision points? What are various cartographic output possibilities resulting at design decision points? To answer these research questions, we have selected the United Nations Gender Inequality Index (GII). This dataset is used to walk through the cartographic design process, reveal different design decisions, and discuss their possible communication outcomes.

The United Nations (UN) has identified seventeen sustainable development goals (SDGs) in an attempt 'to end all forms of poverty, fight inequalities and tackle climate change while ensuring that no one is left behind' by globally measuring and monitoring a consistent set of variables (United Nations, 2016). These SDGs range from eradicating hunger, to access to clean water, to improving the health of the oceans. The aim of the SDGs is to transform how we live today on a global level, shifting policy and practice, with local authorities held accountable to make changes (Fukuda-Parr, 2016). We fully acknowledge that the SDG indicators and indices are far from perfect, but they are useful for the discussion of map-making. SDG indicators that are calculated as part of the GII include maternal health outcomes, adolescent birth rate, a population with at least secondary education, female and male shares of parliamentary seats, and female and male labour force (United Nations Development Programme, 2016).

In an attempt to make a step towards a feminist geography workflow, we reveal problems in the existing dataset, such as missing relevant data, and we question who is and is not included (Moore, 2018; Pavlovskaya \& St. Martin, 2007). We unpack an authoritative dataset assembled by the UN, question it, and offer alternative visualizations thereof, using maps as a tool for fostering discussion. Mapping these data can help elucidate its problems. A map can reveal data inadequacies and support improvements or changes in data collection. It is the first step in a longer process.

We first show how the cartographic process can be blended with a feminist approach to design. As a response to the call for 'doing feminist 
data visualization' (Hill, Kennedy, \& Gerrard, 2016), our aim is to demystify the process of cartography and to address points where a designer makes pivotal design decisions that may alter the narrative of the visualization. By identifying these decision points, we encourage critical thinking and visual literacy associated with cartography whilst drawing attention to data representing women and challenges unique to women. Here we present ways in which the cartographic process, both for a mapmaker and a map user, can adhere to feminist epistemologies. In this chapter, we provide illustrated examples covering data processing, transformation, and visualization to display how design decisions may influence meaning-making. We aim to identify cartographic visualization design and interpretation techniques that foster understanding and inspire action to combat inequities. This responsibility is not only for the mapmaker but also the map-reader.

\section{Gender inequality and the power of cartography}

Gender inequity is a type of injustice that affects and is affected by all and is a significant problem in all regions of the world. When women are supported, so are men, when women have educational opportunities, economic improvement advances for everyone (Duflo, 1997). The causes of social inequities are deeply rooted in complex historic socio-economic challenges that plague entire regions and influence daily life as well as policy and leadership. Maps could help reveal the complex structures that cause inequity by parsing out individual variables, revealing patterns that may otherwise go unnoticed. This conversion from data to information may lead to new insights that can be used to identify appropriate and localized solutions.

Maps and visualizations are thought to be factual, objective, and transparent, but maps are made by people with individual positionalities and epistemology (Elwood, 2009; Kennedy, Hill, Aiello, \& Allen, 2016). Maps often offer both direct and indirect messages with unintended and implicit meanings (MacEachren, 2004). These meanings are conveyed through representations and symbols. Depending on the nature of the data, symbols can vary in appearance. Symbols can vary in size depending on quantities to be represented, or have different colours depending on the quality of the data elements. Colours evoke emotions and hold a different meaning in different cultures. Feelings that emerge from interacting with specific visualizations influence what is learned from them (Kennedy \& Hill, 2017). The use of visual variables, particularly when representing sensitive and important messages associated with underrepresented populations, will influence how and what a map user interprets and/or learns from a map. 


\section{Feminist geography, GIS, and cartography}

Feminist geography is a way of knowing and influencing choices of research methodologies, including how they are implemented (England, 2006). Cartography and GIS have been critiqued by feminist scholars who report that this external vantage point, the all-knowing 'God's eye view' of most maps, that seems to offer the ability to see everything from nowhere (Haraway, 1991) is actually situated (Kwan, 2002b). This God's eye view is seen as authoritative, unquestionable, and objective, yet cartographers know well that the map-making process is filled with decision-making and uncertainty.

Cartography has historically been associated with positivist forms of knowledge production and may help answer questions about what and where. Feminist methodologies are associated with qualitative research and can best answer questions about why and how. Mixed methods approaches to research are recognized as an asset of geographic inquiry (England, 2006). Cartography and information visualization can be used to push a feminist agenda by revealing inequity through the use of the same tools that have (inadvertently or not) perpetuated inequity by omitting women's voices and needs from the map, to begin with (Pavlovskaya \& St. Martin, 2007). It is important for feminist geographers to participate in the development of strategies to give a physical map form to feminist discourse by utilizing GIS to advance feminist practices (Kwan, 2002b). We recognize that using tools associated with scientific cartography alone is not enough; it is still critical to acknowledge what is not in the data, and what is missing from the data and the map (Moore, 2018). We take a seemingly traditional cartographic approach but identify points in this process where ideas from the feminist data visualization framework of D'Ignazio and Klein (2016) can be inserted, as a step towards feminist cartography.

Hill et al. (2016) call for more examination of data visualization to uncover hidden biases and sexist discourses within them. Few have attempted to actually 'do' feminist cartography. Feminist cartographies or GIS are practices using tools associated with quantitative methods to further a feminist presence on the map (Pavlovskaya \& St. Martin, 2007). One example of feminist GIS is visualizing the spatially limited, daily trajectory of a subset of women reflected in a space-time cube map (Kwan, 2002a; Kwan, 1999). Kwan (1999) used a unique network-based GIS method to illuminate that conventional accessibility measures have gender bias because they do not take into account gender roles and expectations. Another example of applied feminist GIS is Stephens's (2013) study of OpenStreetMap (OSM) which reflects the gendered priorities in points of interest contributed through 
Volunteered Geographic Information (VGI). OSM is made up entirely of VGI that form the base map used in many popular location-based services (useful for finding a business or service based on your current location). OSM offers several different classifications and options for brothels yet only one for childcare, but adding or changing the classification of points of interest in OSM requires collective consensus (Stephens, 2013). Positionality and interests are reflected in modern forms of map-making in many ways. Those with the power and technical background to make maps often are unaware of specific injustices to represent on a map. Thus, there is a risk of reproducing and reinforcing inequality that occurs in reality, on the map (Elwood \& Leszczynski, 2013; Leszczynski, 2015).

\section{Open data}

In line with the call for equity in the map-making process, there has been a significant push for transparency in governmental data, particularly spatial data. Open data initiatives aim to have a positive impact on both governance and economic sectors and potentially create an 'open innovation economy through software developers, civic society and participation from residents' (Ojo, Curry, \& Zeleti, 2015, p. 2333). This availability presents new opportunities for civic engagement with data. When raw data are publicly available, skills and specialized training are still required to generate effective maps. Technical skills, hardware, software, and time are required to interact with data. These are resources that those suffering inequities often lack.

Open data offer opportunities to analyse data that those in positions of power also use, to produce maps that display data in different ways, to speak to those in power in a visual language that they already understand. However, 'the master's tools will never dismantle the master's house' (Lorde, 1983, p. 27). In other words, open data could be seen as an attempt to preoccupy the oppressed with the master's concerns (Lorde, 1983) as the data that the government collects and makes available may reflect the government's interests which do not always match the people's. Nonetheless, having open data available, as well as tools to interact with data, presents an exciting opportunity for feminist scholars who wish to do advocacy research. Here we argue that cartographic skills and know-how are more important than ever before. There is an opportunity to inspect open data, to create visualizations, illuminate social disparities, to reflect on whose representation of reality is being revealed, and who is benefiting and who is not, according to a particular data visualization. We will demonstrate that fundamental cartographic principles do not change based on the purpose of a map. 


\section{Visualization choices: Points of reflection during the cartographic workflow}

Maps are produced by following a cartographic workflow (Kraak \& Ormeling, 2010). Figure 24.1 depicts a version of this flow, which is usually split into two connected processes: data analysis and design. Even before the cartographer looks at the data in detail several questions need to be considered. The most important are: What is the purpose of the map, is there a story to be told? Who are the target users and/or other potential users (and can they be involved in the design process)? What is the map use environment? Which type of medium will be used to make the map — static or interactive, paper or digital?

With answers to these questions in mind, the cartographer must consider what is possible to communicate with the available data? Are the data qualitative or quantitative? Quantitative data symbology requires the use of specific perceptual properties to encourage the map-reader to immediately comprehend if the map is representing quantities or numbers. Quantitative data may require proportional perceptual properties that can be communicated by applying visual variables based on size to represent amounts. During each step of the process, the cartographer needs to remain critical and reflective to consider each design and alternative options. This typically requires design understanding and skills. The map user should also be 'skilled' and critical, asking, 'What do I really see? And why does the map look as it does?' Does it privilege a certain viewpoint over another?

Answers to these questions are influenced significantly by both the mapmaker and the map user's positionality. Individual experiences and worldviews will influence what is understood from the visualization made and/or offered. In qualitative research, rigour is tightly connected to reflexivity and positionality (England, 1994; Rose, 1997) because these deeply influence how media are produced and understood. Reflexivity needs to be inserted into cartographic design, questioning each design and interpretation choice along the way.

In Figure 24.1 we summarize a set of questions, which we present to cartography students in classrooms. These questions have to be asked because the tools available to make maps encourage the user to simply push a few buttons using default settings and operations which often result in maps that do not meet the intended communication goals. Not all of the best cartographic design processes or practices are available in popular mapping APIs and software, and to date, machines do not spot the bias that may be caused by using inappropriate visualization choices or that could be found in the data themselves. 


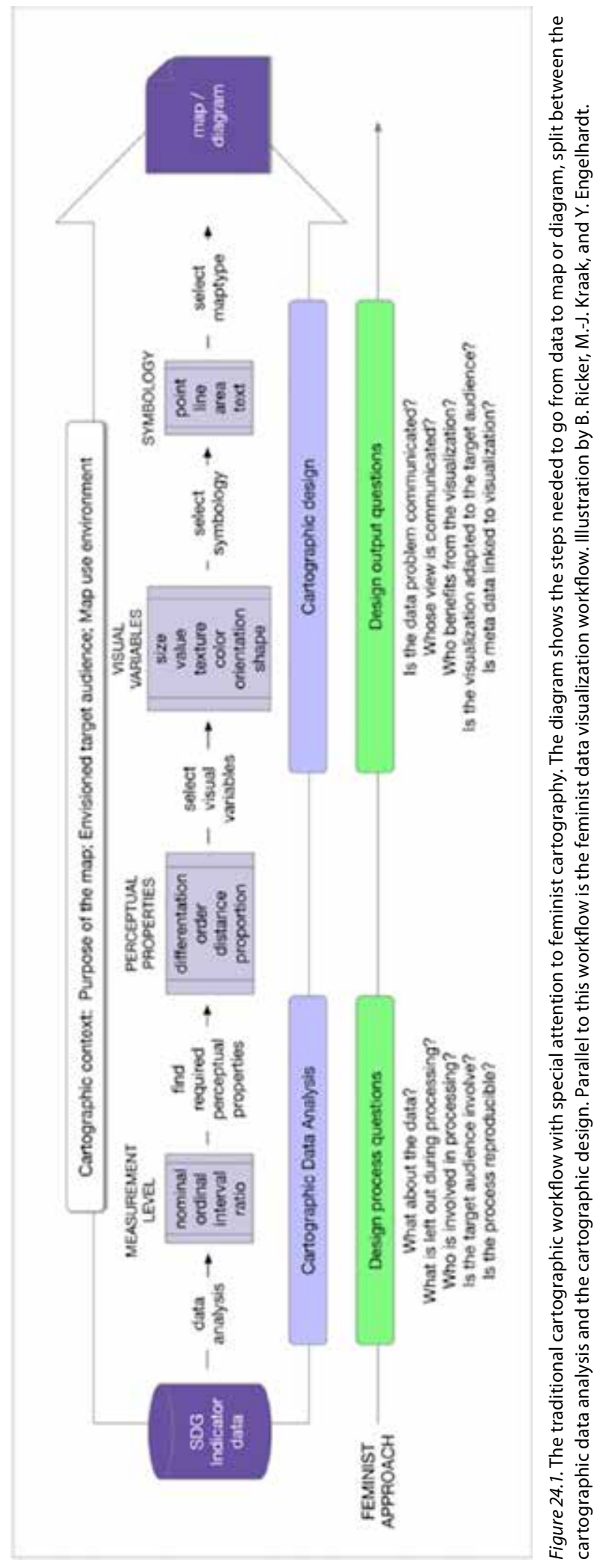


For the purposes of illuminating this workflow, we refer back to the research questions that inform the design process related to the maps we would make using the GII. What is the purpose of the map, is there a story to be told? The purpose of the map is to display the spatial trend of gender inequality as measured and defined by the UN. What is the communication aim? For map-readers to understand what it represents and to critically reflect where GII is a significant problem. Who is the target audience? We see the target users and/or other potential users to be policymakers. What is the map use environment? Which type of medium will be used to make the map-static or interactive, paper or digital? Since this is a book chapter, we decided to make static paper maps. The map use environment is book reading. Next, we start to interact with the data.

Figure 24.2a presents a table with the numbers associated with the GII for each country in alphabetical order. This makes it easy to find a country of interest in the table. Each value is a number between o and 1. In this index o represents no inequality and 1 is maximum inequality. Note that the table does not have an index value for every country. Data in this format are often considered the 'pure' data, but these data are the result of choices that have been made. Here the data have been organized in alphabetical order in English, the number of columns has been chosen, and the index itself is based on choices highlighted in the inset above the bar graph.

In Figure 24.2b, a bar chart offers the same data found in the table, but this time ordered from the highest inequality (left) to lowest (right) for the reader to quickly compare values and countries. Country names are only given every other bar due to space limitations and the countries with no data are omitted. Again, these are examples of choices that have been made; countries have been left out. This does not mean that gender inequality does not exist in these places that lack data.

Figure 24.2c shows a thematic map, a choropleth map that reveals the spatial distribution of GII; in this map the index values have been grouped in four categories based on a particular classification method (natural breaks, which divide classes where there are dramatic differences between low and high points in the data). The visual variable of value has been used to help the user quickly perceive inequality from high (dark) to low inequality (light). Now the countries with the highest inequality are noticed first. This common map type has certain disadvantages. When mapped phenomena are related to the population it is important for the reader to consider that people are seldom homogeneously distributed over the area of the geographic unit (e.g. most of Canada's population lives in a small corridor in the south). 


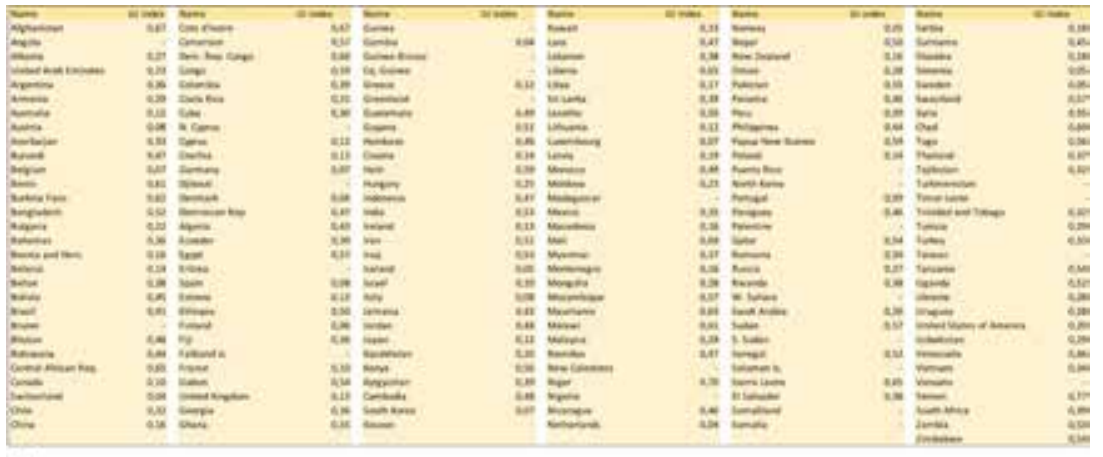

a)
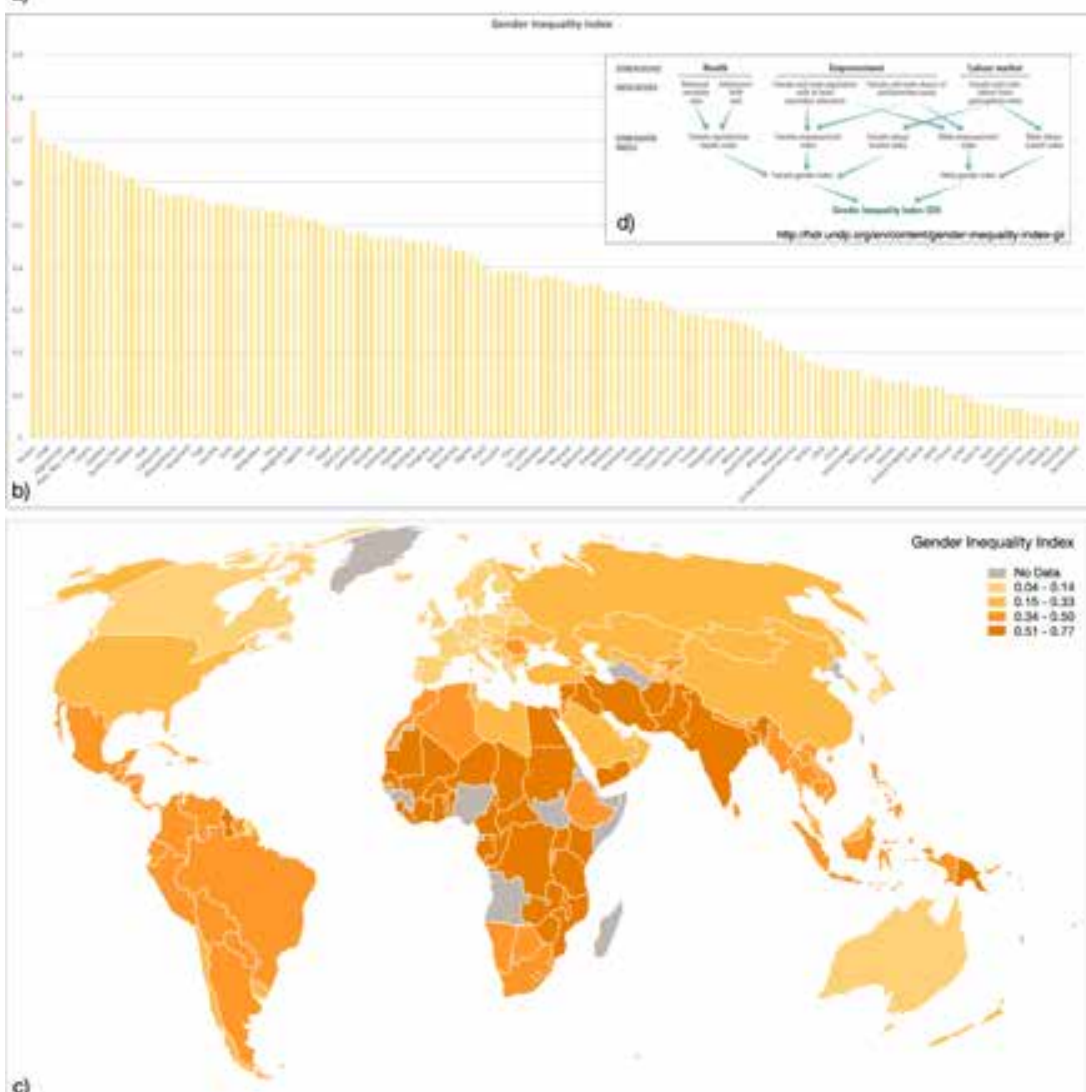

Figure 24.2. The Gender Inequality Index: a) a table with the index value for each country; b) a bar graph with the index order from high to low inequality; c) a map with the geographic distribution of inequality emphasizing high inequality; d) the variables included in the Gender Inequality Index. Source: a), b), c) designed by Ricker, Kraak, and Engelhardt, d) retrieved from http://hdr. undp.org/en/content/gender-inequality-index-gii. 
If we take the feminist geographer's call to read between the lines, and to think about what is missing (Moore, 2018), then gender inequality is considered, valued, and measured differently between individuals, countries, regions, and offices. D'Ignazio and Klein (2016) argue that labour should be made visible. This variable measured in the GII as unpaid labour is a variable within this index, but it is completely unclear how these data are collected. How was the time of unpaid labour measured? Where was it measured? In the context of this research, labour is not visible in terms of who made the maps. According to D'Ignazio \& Klein (2016), this metadata should be recorded and visualized.

Presenting all three graphics in a linked interactive environment would be ideal. The user could select a country in the table, graph, or map, and at the same time see it highlighted in the other graphics.

Following the workflow presented in Figure 24.1 means making many choices with the map objective and targeted audiences in mind. Paying close attention to colour and data classification, we reveal different outcomes of these choices in Figure $24.3 \mathrm{a}-\mathrm{d}$. Each of the four maps presented in Figure 24.3 shows a detailed view of the same data in Africa and Europe. The colour will influence the audience's conclusions. All four maps are choropleth maps. Both maps on the top use a red colour ramp and both maps on the bottom use a green colour ramp. The meaning associated with each of these colours will vary based on the cultural context of the map-reader. Red is typically associated with danger/bad and green is associated with safe/good. With this in mind and with the assumption that low inequality is the optimal situation different patterns emerge. By emphasizing high inequality via the dark red map, Figure 24.3a sends an alert message: this is not good, it is hoped someone will act. If the colour ramp is reversed as in map Figure 24.3 b the problem seems less urgent since the emphasis is on those areas where inequality is not as big a problem. Map Figure 24.3c uses the same approach to the colour ramp as map Figure 24.3a, only now in green. Green does not immediately send an alert, and the mapmaker might downplay the phenomenon. Map Figure 24.3 emphasizes the countries with a low index value. If the maps are there to encourage governments to act, we believe that map Figure 24.3a sends an urgent message. The other maps tell the same story but one has to see through the inverted colour ramps and the colour. The legend shows four classes based on the natural breaks classification. If the number of classes changes or a different classification method were used, the pattern in the map would change dramatically. The audience of any map may not ask, What do I really see? Often a first impression of the map pushes the user to make a particular conclusion. 

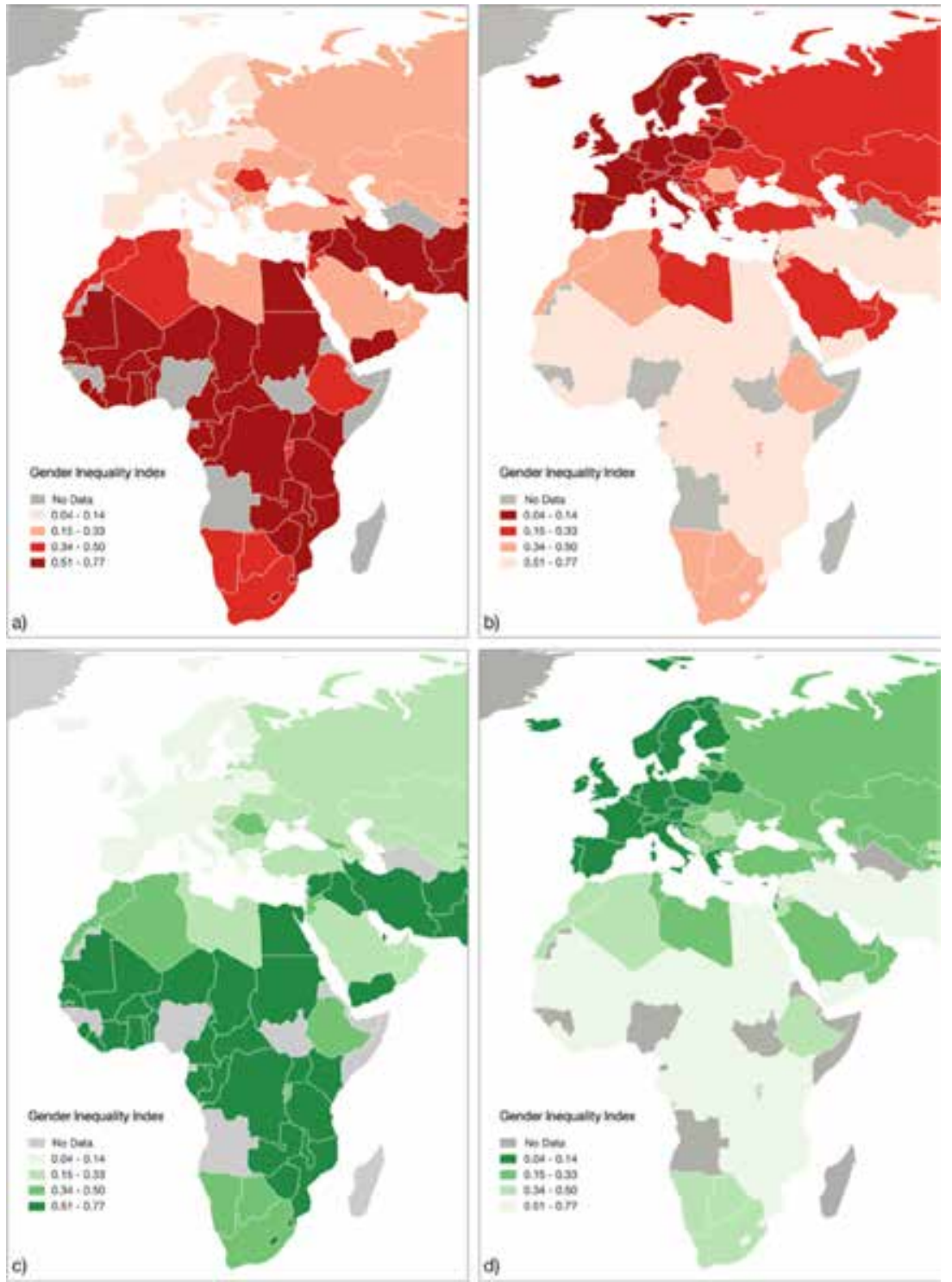

Figure 24.3. Choosing colours and colour ramps for a choropleth map: a) red, emphasizing high inequality; b) red, emphasizing low inequality; c) green, emphasizing high inequality; d) green, emphasizing low inequality. Illustration by Ricker, Kraak, and Engelhardt.

Other key considerations related to the base map selected are the political boundaries and the projection. Borders used in maps convey a specific worldview; lines on maps may cause controversy. Different cartographic techniques, such as blurred or dotted lines, can visualize uncertain and disputed boundaries, but no single map will be able to honour all viewpoints 
regarding any political issue or boundary dispute at a global scale. A decision must be made about which of these techniques most effectively embrace pluralism, multiple views of the world, and consider context (D'Ignazio \& Klein, 2016).

Figure 24.4 shows the influence of the base map, specifically how map projections influence depictions of the world. Figures 24.2, 24.3a-d and 24.4a are all in the Mollweide map projection, an equal area projection. Saudi Arabia (approx. 2.15 million $\mathrm{km}^{2}$ ) and Greenland (approx. 2.16 million $\mathrm{km}^{2}$ ) appear to be roughly the same size in the Mollweide projection. Not all map projections preserve area. In map $23.4 \mathrm{~b}$ the world is projected using the Mercator projection, used by the majority of web mapping applications. Mercator's strength is preserving angles, but not size. Saudi Arabia appears to be far smaller in area than Greenland in map 23.4b. Mercator is an inappropriate projection for a global scale choropleth map since a map user will consider both the representation of colour and the size of the countries. Canada is only slightly bigger than Australia in area (see top map 23.4a), but map 23.4b may suggest a different story: Canada might be evaluated as more significant because it appears much larger in size.

In map $23.4 \mathrm{C}$ a cartogram is presented. In a cartogram, the size of the area shown depends on another variable, in this case the country's population. While comparing maps 23.4a and 23.4c the size of Canada has been reduced and India has been increased. The advantage of this cartogram is that India's increased size shows that more people are being affected by the problem than in Greenland, which looks much bigger in 23.4b.

\section{Conclusion}

Maps evoke emotion, and design choices influence meaning or understanding of maps. Incorporating ideas from feminist GIS, we offered examples of how seemingly simple and arbitrary design decisions may alter what is learned from a map. We displayed common design decisions that cartographers have to make, and in Figure 24.1 we synthesized reflective practice associated with the cartographic process. Based on Figures 24.2-24.4, and connecting them with the design output questions offered in Figure 24.1, we asked: Do we communicate the data problems? Whose view is communicated? Who benefits from the visualization? Do we adapt the visualization to the target audience? Is metadata linked to the visualization? 

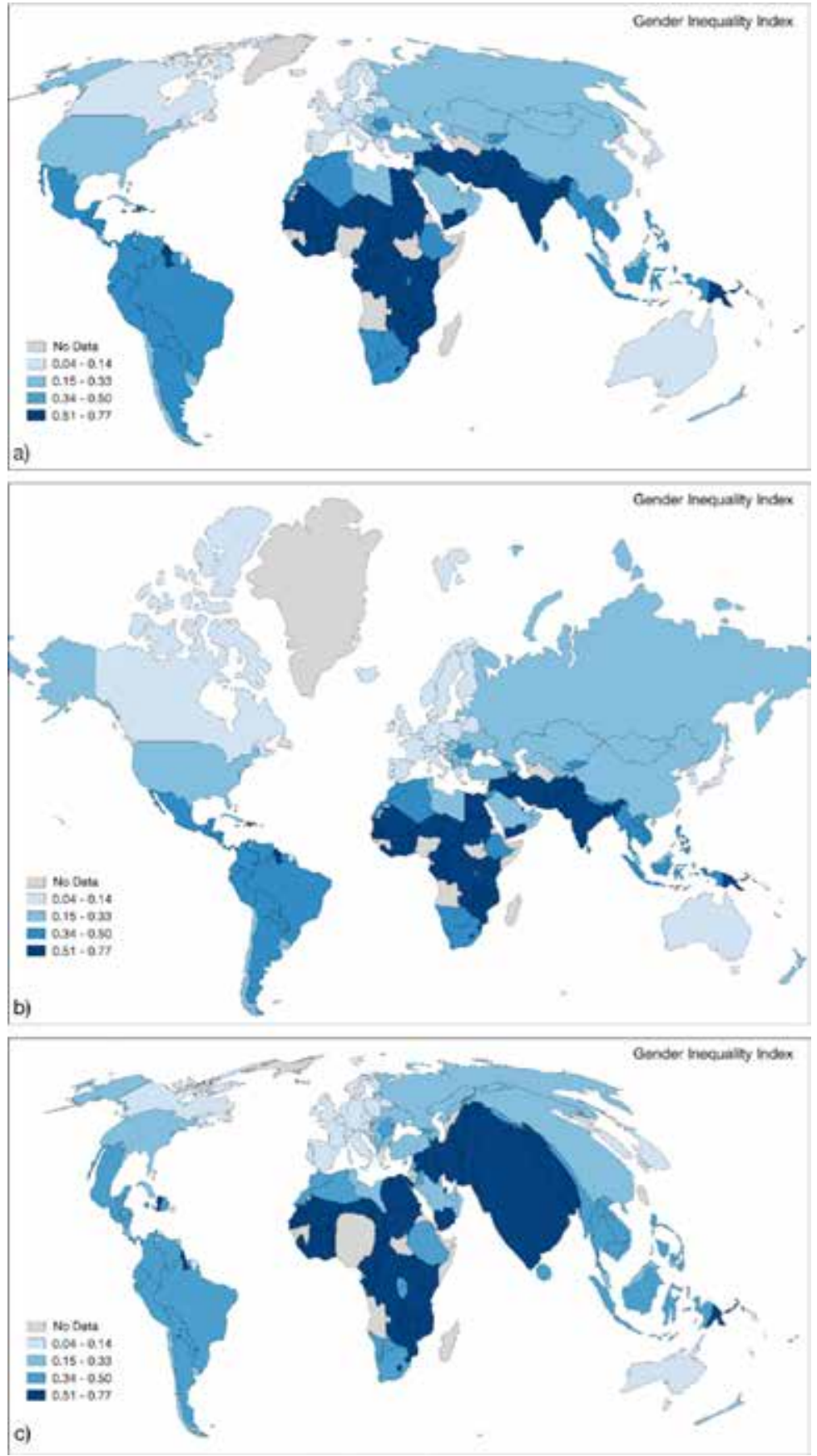

Figure 24.4. The influence of the map projection: a) the equal-area Mollweide projection; $b$ ) the conformal Mercator projection; c) a cartogram based on the country population. Illustration by Ricker, Kraak, and Engelhardt. 
It is well known that cartography is not an objective science. The positionality of the cartographer influences the data interpretation and design decisions, and their subject position will influence the meaning of the map. Maps can be a tool for advocacy, and for this reason, we encourage feminist geographers to use maps as a praxis and part of advocacy efforts to combat and mitigate gender inequality. Our aim is to encourage more people to create visualizations that reflect and reveal women's subject position in an effort to combat and reduce gender inequity. Using a feminist lens, like Kwan (2002a), we see the methods associated with GIS, cartography, and geovisualization as means to identify what type of gender inequity is happening and where it is happening. Future research is needed to then implement qualitative methods to explore the why of these phenomena. As England (2006) points out, qualitative and quantitative methods are not mutually exclusive, particularly in cartography, which is an art and a science.

We acknowledge the critiques of GIS, data visualization, and the SDGs themselves, but we also see an opportunity to use all of these tools together for subversive purposes, to illuminate problems with the data themselves. These could be activists who would like to better understand the spatial distribution of gender inequality as measured by the UN, and challenge these measures. By identifying specific points in the cartographic workflow where design decisions could change outcomes, we aim to encourage critical thinking about and questioning of maps.

Here we echo the call to 'do' more feminist visualization (Hill et al., 2016). Feminist cartography is not a straightforward task. We do not claim to have achieved this end, but we hope these are steps that could precede or complement additional efforts that are taken by feminist geographers. Through mapping open data, what is missing from the data can more easily be identified by a critical map-reader. This can be seen in parallel with 'embrace pluralism' as suggested by D'Ignazio \& Klein (2016) to be open for a different angle on map design and interpretation. Taking this seemingly traditional mapping practice of an authoritative data source could be a step in problematizing the data. This step could be seen as one in feminist geography workflows, to read between the lines and consider what is missing from the data and the map (Moore, 2018; Pavlovskaya \& St. Martin, 2007). We encourage others to utilize open data in an effort to improve data collection, to become a more inclusive practice. We invite more participants to share what they value, to map their worldview. 


\section{References}

D’Ignazio, C., \& Klein, L. (2016, October). Feminist data visualization. Paper presented at and published in the workshop proceedings from the Workshop on Visualization for the Digital Humanities at IEEE VIS Conference, Baltimore, Maryland.

Duflo, E. (1997). Women empowerment and economic development. Journal of Economic Literature, 50(4), 1051-1079. Retrieved September 18, 2019 from http:// www.jstor.org/stable/1049571

Elwood, S. (2009). Geographic Information Science: New geovisualization technologies-emerging questions and linkages with GIScience research. Progress in Human Geography, 33(2), 256-263. https://doi.org/10.1177/0309132508094076

Elwood, S., \& Leszczynski, A. (2013). New spatial media, new knowledge politics. Transactions of the Institute of British Geographers, $38(4)$, 544-559. https://doi. org/10.1111/j.1475-5661.2012.00543.x

England, K. (1994). Getting personal: Reflexivity, positionality, and feminist research. Professional Geographer, 46(1), 80-89. https://doi.org/10.1111/j.00330124.1994.00080.x

England, K. (2006). Producing feminist geographies: Theory, methodologies, and research strategies. In: S. Aitken \& G. Valentine (Eds.), Approaches to human geography. (pp. 286-297). London: Thousand Oaks; New Delhi: SAGE Publications.

Fukuda-Parr, S. (2016). From the Millennium Development Goals to the Sustainable Development Goals: Shifts in purpose, concept, and politics of global goal setting for development. Gender \& Development, 24(1), 43-52. https://doi.org/10.1080/1 3552074.2016.1145895

Haraway, D. (1991). Simians, cyborgs, and women: The reinvention of nature. New York: Routledge.

Hill, R. L., Kennedy, H., \& Gerrard, Y. (2016). Visualizing junk: Big data visualizations and the need for feminist data studies. Journal of Communication Inquiry, 4o(4), 331-350. https://doi.org/10.1177/0196859916666041

Kennedy, H., \& Hill, R. L. (2017). The feeling of numbers: Emotions in everyday engagements with data and their visualisation. Sociology, 52(4), 830-848. https:// doi.org/10.1177/0038038516674675

Kennedy, H., Hill, R. L., Aiello, G., \& Allen, W. (2016). The work that visualisation conventions do. Information, Communication and Society, 19(6), 715-735. https:// doi.org/10.1080/1369118X.2016.1153126

Kraak, M. J., Ormeling, F. (2010). Cartography: Visualization of geospatial data (3rd ed.). Abingdon: Routledge. 
Kwan, M.-P. (2002a). Feminist visualization: Re-envisioning GIS as a method in feminist geographic research. Annals of the Association of American Geographers, 92(4), 645-661. https://doi.org/10.1111/1467-8306.00309

Kwan, M.-P. (2002b). Is GIS for women? Reflections on the critical discourse in the 1990s. Gender, Place, and Culture: A Journal of Feminist Geography, 9(3), 271-279.

Kwan, M. P. (1999). Gender and individual access to urban opportunities: A study using space-time measures. The Professional Geographer, 51(2), 210-227. https:// doi.org/10.1111/0033-0124.00158

Leszczynski, A. (2015). Spatial media/tion. Progress in Human Geography, 39(6), 729-751. https://doi.org/10.1177/0309132514558443

Lorde, A. (1983). The Master's Tools Will never dismantle the Master's house. In: C. Moraga, G. Anzaldúa, T. C. Bambara (eds.), This bridge called my back: Writings by radical women of color. (pp. 94-101). New York: Kitchen Table Press.

MacEachren, A. M. (2004). How maps work: Representation, visualization, and design. New York: Guilford Press.

Moore, F. (2018). Historical geography, feminist research and the gender politics of the present. Geography Compass, 12(9), 1-8. https://doi.org/10.1111/gec3.12398

United Nations. (2018). Sustainable Development Goal indicators website. Retrieved June 28, 2018 from https://unstats.un.org/sdgs/

Ojo, A., Curry, E., \& Zeleti, F. A. (2015). A tale of open data innovations in five smart cities. Proceedings of the Annual Hawaii International Conference on System Sciences, 2015-March, 2326-2335. https://doi.org/10.1109/HICSS.2015.280

Pavlovskaya, M. (2018). Critical GIS as a tool for social transformation. The Canadian Geographer / Le Géographe Canadien, 62(1), 40-54. https://doi.org/10.1111/cag.12438

Pavlovskaya, M., \& St. Martin, K. (2007). Feminism and Geographic Information Systems: From a missing object to a mapping subject. Geography Compass, $1(3)$, 583-6o6. https://doi.org/10.1111/j.1749-8198.2007.00028.x

Pickles, J. (1995). Ground truth: The social implications of geographic information systems. New York: Guilford Press.

United Nations Development Programme [UNDP] (2016). Gender Inequality Index. Retrieved June 28, 2018 from http://hdr.undp.org/en/content/gender-inequalityindex-gii

Rose, G. (1997). Situating knowledges: Positionality, reflexivities, and other tactics. Progress in Human Geography, 21(3), 305-320. https://doi.org/10.1191/ 030913297673302122

Stephens, M. (2013). Gender and the GeoWeb: Divisions in the production of usergenerated cartographic information. GeoJournal, 78, 981-996.

United Nations website (2016). (Retrieved in 2018).

https://www.un.org/sustainabledevelopment/development-agenda/

Wood, D. (1992). The power of maps. New York: Guilford Press. 


\section{About the authors}

Britta Ricker is an Assistant Professor at the University of Twente in the Netherlands. Her research interests focus on accessible spatial technologies, particularly the use of open data and mobile map interfaces.

Menno-Jan Kraak is a Professor of Geovisual Analytics and Cartography and head of the Geo-Information Processing Department at the University of Twente/ITC. He was President of the International Cartographic Association from 2015-2019. He has written more than 200 publications about cartography, geovisualization, and mapping time.

Yuri Engelhardt is an Assistant Professor of Data Visualization at the University of Twente. He is passionate about how visualizing information can contribute to sustainable human well-being. He has written about 'The language of graphics' and 'Diagrams for the masses'. 
